\title{
OPTIMAL ALLOCATION OF INITIAL ATTACK RESOURCES TO MULTIPLE WILDFIRE EVENTS
}

\author{
D. RIDEOUT 1 , Y. WEI ${ }^{1} \&$ A. KIRSCH ${ }^{2}$ \\ ${ }^{1}$ Department of Forest, Rangeland and Watershed Stewardship, Colorado State University, USA. \\ ${ }^{2}$ National Park Service, USA.
}

\begin{abstract}
Increased scrutiny of federally funded programs combined with changes in fire management reflects a demand for new fire program analysis tools. We formulated an integer linear programming (ILP) model for initial attack resource allocation that operates in a performance-based, cost-effectiveness analysis (CEA) environment. The model optimizes the deployment of initial attack resources for a user-defined set of fires that a manager would like to be prepared for across alternative budget levels. The model also incorporates fire spread, multiple ignitions, simultaneous ignitions, and monitoring of resources on a landscape. It also evaluates the cost effectiveness of alternate firefighting resources and alternative pre-positioning locations. Fires that escape initial attack are costly during the extended attack phase of fire management. To address this within the scope of initial attack, we constructed and analyzed alternative objective functions that incorporate a proxy for internalizing the cost of fires that escape initial attack. This type of model can provide the basis for a wider scale formulation with the potential to measure an organization's performance and promote a higher level of accountability and efficiency in fire programs.

Keywords: fire escape, initial attack, integer linear programming, optimal deployment, performance, wildland fire.
\end{abstract}

\section{INTRODUCTION}

In the United States, the major federal land management agencies conduct an extensive planning and budgeting analysis to prepare for the upcoming fire season(s). Historically, these agencies have used varied and often dissimilar analytical models and approaches to prepare the annual budget and its allocation to the planning units responsible for wildland fire protection. These historical efforts have come under scrutiny in recent years as preparedness budgets and the need for accountability have increased dramatically. The U. S. General Accounting Office (GAO) urged federal land management agencies to develop a framework that will ensure the effective use of billions of dollars set aside for wildland fire activities [1]. The GAO continues to highlight the need for cost-effective wildland fire spending and specifically preparedness spending [2]. A central part of preparedness planning is the preparation for initial attack activities that often involves initial attack modelling.

Wildland fire organizations, including U.S. federal land management agencies, customarily organize the suppression of unwanted fires into the three stages of suppression: initial attack (IA), extended attack (EA), and large fire management. Compartmentalizing this problem allows organizations to focus on the functioning and funding of different stages of fire management. This enables the analyst to focus with depth on the part of the problem of primary interest, but it introduces the problem of potential costly 'spill over' effects. For example, initial attack fires that are not contained will spill over into extended attack or even to large fire management. The potential cost of such spillover is a necessary consideration in a proper benefit and cost calculus of initial attack.

The wildland fire management literature includes simulation and optimization methods to address various parts of the wildland fire management programs. Simulation used in 
preparedness planning includes models such as the Fire Economics Evaluation System (FEES) [3], the National Fire Management Analysis System (NFMAS) [4], the California Fire Economics Simulator 2 (CFES2) [5], Level of Protection Analysis (LEOPARDS) [6], and Wildfire Initial Response Analysis System (WIRAS) [7]. These models have important strengths in their ability to simulate the effects of a particular set of firefighting resources and some have been used to help managers evaluate initial response problems.

While simulation modelling has been fruitful, especially in showing effects of a given set of resources, optimization enables managers to focus on strategic elements of initial attack such as identifying the optimal set(s) of firefighting resources for a planning unit, or for modeling resource allocation across multiple fire events. Parks [8] designed a deterministic model to minimize the cost of suppression plus loss to identify an optimal constant workforce. Parlar and Vickson [9] and Parlar [10] extended the Parks' model using optimal control theory. Aneja and Parlar [11] extended Parks' model using nonlinear programming to estimate optimal staffing of a firefighting organization by minimizing the cost plus loss per unit time. Boychuk and Martell [12] evaluated seasonal forest firefighter requirements with Markov chains utilizing the least cost plus loss framework. Donovan and Rideout [13] used ILP to optimize a firefighting resource allocation to a single fire using a cost plus net value change framework. Haight and Fried [14] used a scenario-based standard response model as an extension of the classic maximal covering location model. Bevers [15] proposed the potential for using stochastic programming and chance constraint to address the uncertainties in fire management.

We build on previous optimization literature by using a natural extension with modifications of the Donovan and Rideout [13] formulation to address the issues of multiple fires, simultaneous fires, monitoring resources, with special attention to potential spillover effects from IA to EA. A demonstrative example shows how an ILP model can be used to identify and optimize the dispatch of initial response resources in a performance-based and cost-effectiveness analysis (CEA) framework. The analysis includes four important features that have not been previously demonstrated: (i) use of an integer linear program (ILP) to model a functional relationship between cost and performance, (ii) inclusion of multiple fires and optimal dispatch locations, with the potential to address a season of fires, (iii) the capability of including simultaneous ignitions, and (iv) because fires that escape initial attack can be costly, we address alternative means of including a proxy for the cost of fires that escape initial attack. The remainder of the paper is structured as follows: in the next section we present a description and a mathematical formulation of the ILP with alternative objective functions to address the cost of escaped fires, followed by a demonstrative numerical example to illustrate the capabilities and relationships of the model. The last section provides discussion and conclusions including model limitations and potential extensions of the formulation.

\section{A PERFORMANCE-BASED FIRE-PREPAREDNESS ILP}

We make the customary assertion of minimizing damage for a given level of expenditure consistent with the least cost plus loss expressions [16]. Consistent with this assertion, we compare the effectiveness of alternative initial attack organizations by minimizing expected damage (loss) of unwanted wildland fires for any specified budget level where a range of budget levels are modeled. We recognize that to the extent that firefighting resources are scarce, not all fires are of equal importance to contain because not all resources that could be damaged by fire are of equal consequence. Wildland fires occurring in the wildland urban interface threaten life and property. They are typically of greater importance to aggressively 
manage than are fires occurring in remote areas such as wilderness. Because acres differ in their importance to protect from wildfire, our formulation provides the ability to proportionally weigh acres that might be differentially affected by the damaging effects of wildfire [17]. The calculation of natural resource loss for a given budget level involves multiplying the area burned from each fire by its per acre weight to calculate the per acre loss. The weight reflects the marginal rate of substitution of resource disimprovement. The ILP optimization allows us to focus on cost-effective solutions while avoiding interior (inferior) solutions.

When preparing for a wildfire season, managers can predict their prospective fire situation. For example, through the use of geographical information systems, managers can accurately map locations, conditions, and types of fuels that can be used to describe fire activity across the planning unit. Managers can estimate the set of conditions they would like to plan for including a set, or sets, of fires to model using prediction and forecasting models [18-21]. Such data can portray current and future landscape conditions. Predictive tools can be used to develop a fire scenario(s) for which a manager would like to be prepared.

This set of fires is provided as input to the ILP and each fire includes information on its initial size and its change in perimeter and area by time period. Perimeter is directly related to suppression cost through resource production rates and the area burned is directly related to performance through expected loss. Other fire behavior characteristics such as flame length and fire intensity can be reflected in the firefighting resources' ability to build fireline, which allows managers to incorporate tactical firefighting standards: for example, a fire with flame lengths of 4 to 8 feet can be too intense for a direct attack with hand tools, but bulldozers, engines, and aerial drops might be effective [22].

We use the free burning fire containment rule from previous deployment models ( e.g. see USDA Forest Service [4] and Donovan and Rideout [13]) stating that a fire is contained when the total fireline produced by firefighting resources overtakes the fire perimeter. A fire is defined as having 'escaped' if it is not contained during the initial attack period due to a lack of funds to apply to firefighting resources resulting in a lack of sufficient fireline production capability.

A pool of potential firefighting resources is established for evaluation where each resource can be selected and optimally allocated to a set of candidate dispatch locations and fire events. Each firefighting resource is defined by a fireline production rate and by its fixed and variable costs. Fireline production is modeled by a cumulative value that is input for each time step of each fire. An advantage of the discrete time step approach is that the production function does not have to be constant or linear. Thus, production rates can reflect fatigue and other disruptions in production such as water and fuel refills. Arrival times and travel delays can also be reflected in these production values by entering zero chains of fireline production during travel periods. The model uses the production information along with other factors to solve for the optimal deployment.

The costs of initial response resources and of fire escapes are important considerations in preparedness modeling that directly impact the preparedness budget. This ILP model inputs fixed and variable costs of firefighting resources that directly impact optimal deployment. The fixed cost is modeled as a one-time charge that is incurred if the resource is deployed to any fire during the season. Each resource's variable cost is modeled as an hourly cost that reflects its operating expenses on each fire including maintenance, fuel, regular hourly wages, overtime, and hazard pay. Also during the IA period, we deploy a monitoring resource to escaped fires to reflect the concept that every fire, contained or not, will receive some monitoring efforts during initial attack. The full cost of escapes is addressed in the section 'Incorporating a Proxy for the Cost for Escaped Fires'. 
Formulating the ILP for fire suppression requires developing a set of equations to track containment on each fire. The Donovan and Rideout [13] ILP optimized firefighting resource allocation to a single fire to minimize the total suppression cost plus net value change. They used a separate set of constraints at each time period to track whether the targeted fire would be contained during that period. This formulation expands their approach to support IA firefighting resource allocations across multiple and simultaneous ignitions. Although firefighting resources can be dispatched to multiple fires, they often cannot be dispatched to simultaneous fires, and this introduces heightened competition for firefighting resources. To model simultaneous ignitions, we forced each resource to choose one of the simultaneous ignitions to attack and we assumed that resources would not be redeployed to other simultaneous ignitions. This restriction reflects the pragmatic consideration that ground-based resources often lack the mobility to address simultaneous fires. We also introduced constraints across time to track the period that each fire was contained.

\section{Mathematical Formulation}

\section{Minimize Loss}

$$
\text { Loss }=\sum_{i} \sum_{d \in\left(1 \text { to } D_{e}\right)}\left(W_{i d} * f_{i d} * A_{i d}\right)
$$

\section{Subject to:}

$$
\begin{array}{cc}
\sum_{k \in K_{r}} u_{r k} \leq 1 & \forall r \\
\sum_{d \in\left(1 \text { to } D_{e}\right)} x_{i r k d} \leq u_{r k} & \forall i, r, k \in k_{r} \\
\sum_{d \in\left(1 \text { to } D_{e}\right)} f_{i d}=1 & \forall i \\
\sum_{r} \sum_{k \in K_{r}} \sum_{d \in(1 \text { to D })}\left(x_{i r k d} * L_{i r k d}\right) \geq \sum_{d \in(1 \text { to D })} f_{i d} * P_{i d} \\
\sum_{d \in(1 \text { to D })} d^{*} f_{i d} \geq \sum_{d \in(1 \text { to D })} d^{*} x_{i r k d} & \forall i, r, k \in k_{r} \\
\sum_{i} \sum_{r} \sum_{k \in K_{r}} \sum_{d \in\left(1 \text { to } D_{e}\right)}\left(x_{i r k d} * H_{r d}\right)+\sum_{r} \sum_{k \in K_{r}}\left(u_{r k} * F_{r k}\right) \leq B \\
\sum_{i \in s_{n}} \sum_{d \in\left(1 \text { to } D_{e}\right)} x_{i r_{g} k d} \leq u_{r_{g} k} \\
\sum_{r} \sum_{k \in K_{r}} x_{i r k D_{e}} \geq f_{i D_{e}} & \forall n, r_{g}, k \in k_{r_{g}}
\end{array}
$$

$i \quad$ index of a fire in the set $I$ of all fires,

$r \quad$ index of a firefighting resource in the set $R$ of all available resources; $r_{g}$ is the index for ground-based resources that cannot move easily between simultaneous fires for IA,

$k \quad$ index of an optional dispatch point in the set $K_{r}$ of all potential dispatch points for resource $r, K_{r}$ denotes the set of potential dispatch points for ground-based resources, index for a group of simultaneous fires, index of a fire in the set $S_{n}$ of the $\mathrm{n}^{\text {th }}$ group of simultaneous fires, $S n \subseteq I$, 
$d \quad$ index of fire durations in the set $D$ and $D_{e} . D$ is the set of periods before a fire escape. $D_{e}$ is the period at which fire is considered to have escaped,

$x_{\text {irkd }}$ binary variable, $x_{i r k d}=1$ if resource $(r)$ allocated at dispatch point $(k)$ is deployed for a duration of $(d)$ time periods to contain fire $(i)$, otherwise $x_{i r k d}=0$,

$f_{i d} \quad$ binary variable, $f_{i d}=1$ if fire $(i)$ burns for a duration of $(d)$ time periods, otherwise $f_{\text {id }}=0$,

$u_{r k} \quad$ binary variable, $u_{r k}=1$ if resource $(r)$ is allocated at dispatch point $(k)$, otherwise $u_{r k}=0$,

$F_{r k} \quad$ the fixed cost of allocating resource $(r)$ at dispatch point $(k)$,

$H_{r d} \quad$ cost accrued for resource $(r)$ for deployment duration of $(d)$ time periods,

$L_{i r k d} \quad$ total (cumulative) line produced on fire $(i)$ by resource $(r)$ allocated at dispatch point $(k)$ for a duration of $(d)$ time periods. This allows the model to test the efficiency of alternative dispatch locations for a particular resource. A particular resource could produce different amounts of fireline on a particular fire because travel distances (time) can differ,

$W_{i d} \quad$ predicted fire losses for each unit of area burned by fire $(i)$ after a duration of $(d)$ time periods,

$P_{i d} \quad$ predicted burn perimeter for fire $(i)$ after a duration of $(d)$ time periods,

$A_{i d} \quad$ total area burned by fire ( $\left.i\right)$ for the duration of $(d)$ time periods,

$B$ the upper bound of initial attack cost input to the model.

The objective function, eqn (1), minimizes the expected fire loss for a given budget. For each firefighting resource, eqn (2) restricts its allocation to a single location. This expands the model to consider alternative locations for any particular resource. Equation (3) restricts each suppression resource $r$ to only be deployed to each fire for a fixed duration. Equation (4) defines the constraint set requiring each fire $f$ to last for a defined duration. Equations (5) and (6) are constraints that define the conditions of successful containment for each fire. For each contained fire, eqn (5) requires that the total length of fireline produced by all suppression resources from different dispatch points must equal or exceed the fire perimeter at the period it is contained. Constraint (6) ensures that fireline will be effective only during the containment period of any fire. The index of fire duration $d$ is used to make this assumption valid in the model. For example, if there is a single suppression resource $r$ ' available of constructing fire line to contain a fire within an $8 \mathrm{~h}$ IA period, this constraint will take a simplified form of:

$$
f_{r^{\prime} 1}+2 f_{r^{\prime} 2}+3 f_{r^{\prime} 3} \ldots \ldots+8 f_{r^{\prime} 8}>=x_{r^{\prime} 1}+2 x_{r^{\prime} 2}+3 x_{r^{\prime} 3} \ldots \ldots+8 x_{r^{\prime} 8}
$$

For example, if this fire lasts two hours before being contained by resource $r^{\prime}\left(f_{r^{\prime} 2}=1\right.$, and all other $f_{r^{\prime} d}=O$ from constraint (4)), only fire line constructed during those two hours counts as effective $\left(x_{r^{\prime} 3}\right.$ through $x_{r^{\prime} 8}$ must each equal 0 and either $x_{r^{\prime} 1}$ or $x_{r^{\prime} 2}$ would equal one). Constraint (7) states that the total cost of all resources deployed to all fires (hourly and fixed), must be less than or equal to the budget $(B)$. This constraint can be used to analyze tradeoff between budget level and resource loss. Equations (8) are used to restrict ground resources from fighting simultaneous fires. Aerial resources such as air-tankers address simultaneous fires and would not be subject to this constraint. Equations (9) reflect the cost of gathering information on fires that may not be contained during the IA period by ensuring that at least one resource is deployed to each uncontained fire. 


\subsection{Incorporating a Proxy for the Cost of Escaped Fires}

While compartmentalizing suppression into IA and EA provides managerial clarity for planning, budgeting, and operations, it introduces a classic externality problem if not properly addressed. In the IA preparedness planning context, such an externality can be generated if the costs of fires that escape IA are not considered in the IA model or decision process. A correct approach, consistent with the Coase Theorem [23], would be to maximize the sum of the net benefits across both program components (IA and EA) when considering resource allocations to IA preparedness planning. Simultaneously modeling both would, in principle, provide the correct set of costs to the IA analysis. In this way we could solve for the optimal number of escaped fires. The problem is that there is no precedent for modeling large fires in this context or for modeling IA and EA simultaneously.

In lieu of a credible simultaneous solution, we tested three potentially practical proxies for the cost of escaped fires by using three alternative objective functions. These were: (i) using a large per escaped fire penalty, (ii) increasing the penalty for escapes in proportion to estimated loss at the time of escape, and (iii) combining approaches one and two. The objective function is separated into two parts where the first part represents the loss during IA and the second part represents a penalty for escapes. Of particular interest is how modifying the second part of the objective function will influence the allocation of IA resources and fire containment.

Objective function (11) penalizes each escaped fire by using a large constant penalty ' $M$ '.

$$
\text { Minimze } \quad \sum_{i} \sum_{d \in(1 \mathrm{to})}\left(W_{i d} * f_{i d} * A_{i d}\right)+M * \sum * \sum_{i} f_{i D_{e}}
$$

As $\mathrm{M}$ becomes large, this objective function effectively maximizes the number of fires contained, regardless of their importance. This is also known as initial attack success rate: a common performance metric.

In objective function (12), escaped fires are penalized by a value proportional to their loss just before escape.

$$
\text { Minimze } \quad \sum_{i} \sum_{d \in(1 \text { to D })}\left(W_{i d} * f_{i d} * A_{i d}\right)+\Omega * \sum_{i}\left(W_{i d} * f_{i D_{e}} * A_{i d}\right)
$$

The penalty increases linearly with respect to loss and the term $\Omega \geq 1$ enables us to increase the magnitude of the penalty. The rationale for penalizing escapes based upon the estimated loss at the time of escape is that it reflects the last information known to the IA model regarding the potential resource damage from an escape. It also reflects the restriction of the scope of the problem to IA preparedness.

Objective function (13) combines (11) and (12) to penalize escapes by using a constant penalty combined with the estimated loss prior to escape. The rationale for adding the per fire escape cost is that escaped fires can be costly to manage even if there is little potential for resource loss at the time of escape.

$$
\text { Minimze } \quad \sum_{i} \sum_{d \in(1 \text { to D })}\left(W_{i d} * f_{i d} * A_{i d}\right)+\Omega * \sum_{i}\left(W_{i D} * f_{i D_{e}} * A_{i D}\right)+M * \sum_{i} f_{i D_{e}}
$$

With the loss minimizing ILP formulated and expressed through three alternative objective functions to address the cost of escapes, we apply the model to a demonstrative example that is designed to show how the model addresses optimal placement and dispatch of resources in a CEA context at different budget levels. 


\section{DEMONSTRATIVE EXAMPLE}

We begin by defining a fire scenario that includes 10 fires $\left(\mathrm{F}_{1}\right.$ through $\left.\mathrm{F}_{10}\right)$ where two, $\mathrm{F}_{9}$ and $\mathrm{F}_{10}$, occur simultaneously (Table 1 ). For simultaneous ignitions we make the simplifying assumption that no single suppression resources can be assigned to both. This assumption can be relaxed to allow some resources to serve simultaneous fires, but such relaxation does not add to the substance of our findings or formulation. We also assume eight time periods where each period is one hour. The duration can take any time step and the time steps are not required to be uniform. The initial perimeter of each fire represents the size of each fire when at discovery and the perimeter of each fire will grow as defined by the user during the eight hour IA period (Table 1).

Table 2 displays the assumed loss for each fire during each of the $8 \mathrm{~h}$ periods with no suppression effort. Because we are using the free burning fire containment rule, any fire shape

Table 1: Fire attributes used in predicting the fire spread in the demonstrate example.

\begin{tabular}{lcc}
\hline Fire name & Initial perimeter (chain) & $\begin{array}{c}\text { Rate of change in perimeter } \\
\text { (chain/h) }\end{array}$ \\
\hline $\mathrm{F}_{1}$ & 8 & 9 \\
$\mathrm{~F}_{2}$ & 24 & 21 \\
$\mathrm{~F}_{3}$ & 19 & 11 \\
$\mathrm{~F}_{4}$ & 9 & 16 \\
$\mathrm{~F}_{5}$ & 15 & 14 \\
$\mathrm{~F}_{6}$ & 23 & 10 \\
$\mathrm{~F}_{7}$ & 19 & 20 \\
$\mathrm{~F}_{8}$ & 11 & 12 \\
$\mathrm{~F}_{9}$ & 30 & 7 \\
$\mathrm{~F}_{10}$ & 15 & 17 \\
\hline
\end{tabular}

Table 2: Expected loss by each fire at each period without initial attack. Fires F9 or F10 are assumed to occur simultaneously.

\begin{tabular}{|c|c|c|c|c|c|c|c|c|}
\hline \multirow[b]{2}{*}{ Fire name } & \multicolumn{8}{|c|}{ Expected fire loss at each period P1 through P8 } \\
\hline & $\mathrm{P} 1$ & $\mathrm{P} 2$ & P3 & $\mathrm{P} 4$ & P5 & P6 & P7 & P8 \\
\hline $\mathrm{F}_{1}$ & 0.1 & 2.0 & 6.8 & 13.7 & 25.2 & 54.4 & 76.9 & 119.0 \\
\hline $\mathrm{F}_{2}$ & 2.5 & 13.3 & 36.5 & 98.0 & 219.2 & 315.8 & 564.4 & 765.6 \\
\hline $\mathrm{F}_{3}$ & 2.0 & 11.9 & 34.9 & 59.2 & 117.8 & 163.9 & 251.8 & 338.2 \\
\hline $\mathrm{F}_{4}$ & 1.4 & 12.5 & 41.1 & 88.2 & 155.7 & 233.6 & 361.9 & 566.8 \\
\hline $\mathrm{F}_{5}$ & 1.6 & 6.2 & 26.1 & 49.1 & 101.3 & 179.6 & 284.7 & 371.5 \\
\hline $\mathrm{F}_{6}$ & 2.8 & 11.5 & 20.8 & 40.2 & 62.1 & 99.6 & 134.1 & 174.4 \\
\hline $\mathrm{F}_{7}$ & 0.5 & 4.3 & 17.9 & 32.5 & 61.9 & 147.1 & 229.0 & 429.7 \\
\hline $\mathrm{F}_{8}$ & 0.1 & 2.0 & 17.8 & 36.8 & 126.1 & 280.1 & 385.3 & 558.6 \\
\hline $\mathrm{F}_{9}$ & 2.8 & 6.7 & 11.4 & 19.8 & 30.9 & 43.3 & 63.9 & 92.4 \\
\hline $\mathrm{F}_{10}$ & 1.8 & 8.4 & 20.3 & 64.9 & 131.4 & 191.6 & 314.4 & 513.7 \\
\hline
\end{tabular}


could be chosen, but we chose a standard 2:1 ellipse [24]. We calculate the area burned for each period based on the initial fire size and growth rate of each fire listed in Table 1. If the fire is not contained within the eight hours allocated for IA, it was defined as 'escaped' and a resource was deployed to monitor the fire for the IA period as some cost and effort will be expended during the IA period.

Our list of firefighting resources was selected to illustrate key model features of optimal allocation and dispatch while recognizing that agency planning units would be considerably more complex. For demonstration we modeled three kinds of resources: resources that are relatively inexpensive and have relatively low production rates such as handcrews, resources that are moderately expensive but produce greater line production such as engines, and we also included dozers as an expensive and highly productive resource. Resource production rates were based on the National Wildfire Coordination Group Fireline Handbook [25].

To demonstrate the model's ability to evaluate optimal resource placement, we allowed the model to choose from three possibilities: dispatch from location HC1.A, dispatch from location HC1.B, and no dispatch. With such choices, the model deploys the most economically efficient set of resources to aid with the selection of locations. Firefighting resources that are not dispatched from a given location, do not arrive, incur no cost, and produce no line. The difference in dispatch locations is represented by differences in arrival times and by the subsequent fireline production on each fire. The cost and productivity of each kind of resource is listed in Table 3. By using the data from the tables on fire growth, expected loss, and firefighting production, we generated the following results.

\section{RESULTS}

The results of the model formulation using the demonstrative example are discussed in two parts: (i) model formulation on resource allocation and fire containment with the effects of simultaneous fire events including the use of monitoring resources, and (ii) effects of the alternative objective functions reflecting different proxies for the cost of escaped fires.

Table 3: The cost of firefighting resource and their production rates of fire line construction.

\begin{tabular}{lccc}
\hline Firefighting resource & Fixed cost $(\$)$ & Hourly cost $(\$)$ & $\begin{array}{c}\text { Line production rate } \\
\text { (chain/h) }\end{array}$ \\
\hline HC1.A* & 2,050 & 250 & 9 \\
HC1.B* & 2,050 & 250 & 9 \\
HC2 & 2,030 & 250 & 9 \\
HC3 & 1,000 & 100 & 3 \\
$* *$ Eng1 & 8,000 & 400 & 16 \\
Eng2 & 8,500 & 400 & 16 \\
Eng3 & 5,000 & 300 & 12 \\
Dozer & 18,000 & 900 & 30 \\
\hline
\end{tabular}

* Handcrew 1 (HC1) can be located at either dispatch point A or B with different arrival times to each fire.

*'Eng' represents 'Fire Engine'. 


\subsection{Resources and fires}

The detailed containment period for each fire and the allocation and dispatch schedule for each resource are shown in Table 4 based on a budget level of \$21M. At this budget level all fires can be contained and there was no difference among the alternative objective functions for escaped fire cost. Also, as shown in Table 4, the deployment duration of any resource is less than or equal to the duration of each corresponding fire. This is consistent with constraints (5) and (6). The necessary and sufficient condition of containing fire $i$ at period $d$ is that the total length of fireline produced for fire $i$ at or before period $d$ has to be equal to or longer than the perimeter of fire $i$ at period $d$.

All fires, except for the simultaneous fires $\left(\mathrm{F}_{9}\right.$ and $\left.\mathrm{F}_{10}\right)$ were contained within the first or the second hour. The key advantage to containing fires earlier is to reduce potential loss. Keeping fires small also means that less fireline is needed and this should not imply a lower suppression cost because minimizing fire size implies an intensive effort that could employ the most expensive and productive equipment and labour. The results also show that handcrew 1 would be allocated to dispatch point B at this budget level and that handcrews 2 and 3 and engine 3 were also dispatched. The expensive and technically superior dozer was not dispatched at this budget level.

The ILP was required to make 'tough' choices in resource deployment on the simultaneous fires. Fire $\mathrm{F}_{10}$ used all of the hand crew resources while fire $\mathrm{F}_{9}$ relied entirely upon engine three. The opportunity cost of deploying all of the handcrews to $\mathrm{F}_{9}$, in terms of reduced effectiveness on $\mathrm{F}_{10}$, is apparent as it took longer to contain $\mathrm{F}_{9}$ (five time periods). The cost of deployment includes both the variable cost of deploying the resource plus the opportunity cost incurred by not allowing that resource to attack the competing simultaneous fire. Additional tests showed that after removing the assumption of simultaneity for fires $\mathrm{F}_{9}$ and $\mathrm{F}_{10}$, a $100 \%$ IA success rate was achieved at a lower budget level of $\$ 18 \mathrm{M}$.

\subsection{Alternative proxies for the cost of escaped fire}

Results from the model at the 11 different budget levels were used to produce the cost effectiveness frontiers in Fig. 1a. Each point on the frontier corresponds with a unique deployment of resources that minimizes the loss during IA at the specified budget level. Three frontiers were produced on the basis of the objective functions (11), (12), and (13) where $\Omega=1$ in (12) and (13).

Our tests of the 11 budget levels show that all 10 fires can be contained at budgets of $\$ 21 \mathrm{M}$ or above within the $8 \mathrm{~h}$ initial attack period (Fig. 1b). Fire containment schedules are insensitive to the choice of objective function above this budget level. Reducing the budget increased scarcity and the model allowed some fires to escape. The number of escapes was affected by the budget level and the objective function. For a given budget level, which fires escaped was sensitive to how the proxy cost of escapes was modeled.

Analysis of objective function (11) showed that the model would contain as many fires as possible (Fig. 1b). That is, a simple per fire proxy for the cost of escapes maximized initial attack success rate. This objective function will always maintain or increase the number of contained fires with increases in the budget. However, using this kind of objective function produces dispatch schedules with higher fire losses during IA (Fig. 1a). Because its constant penalty treats all fires with equal importance for containment, it fails to recognize the relative importance between fires. For example, the results show that with a budget level of $\$ 20 \mathrm{M}$, the model could contain either $F_{9}$ or $F_{10}$ at period two, but not both of them. 


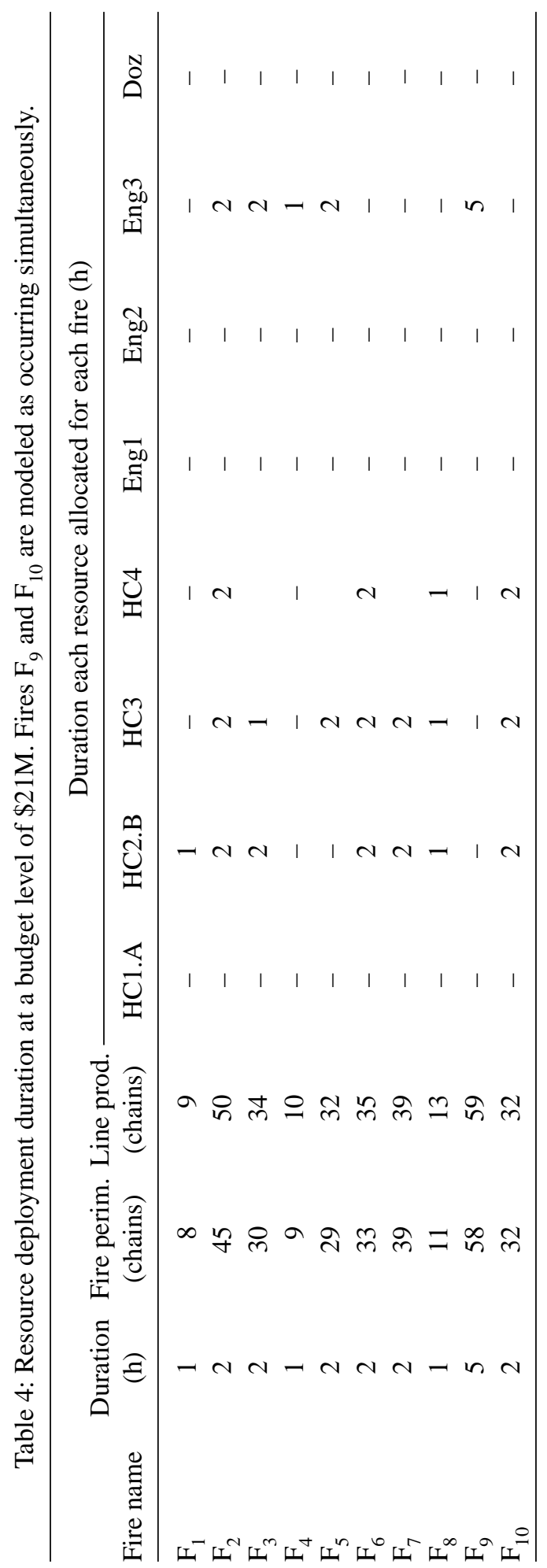




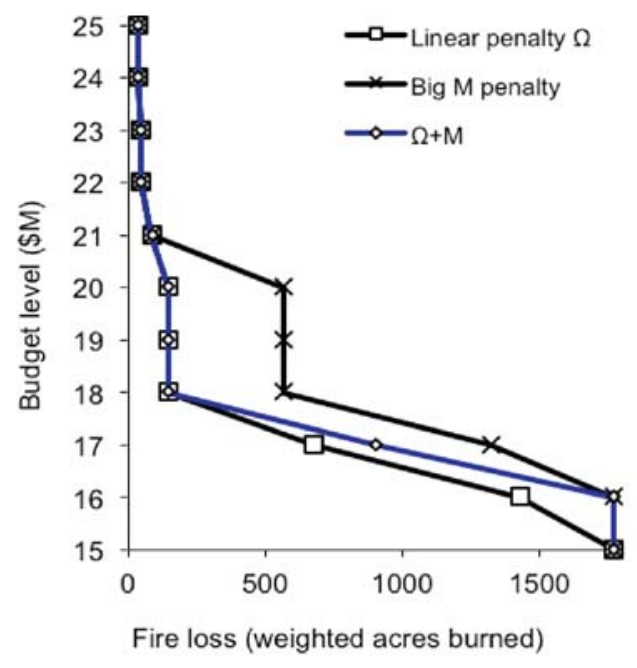

(a)

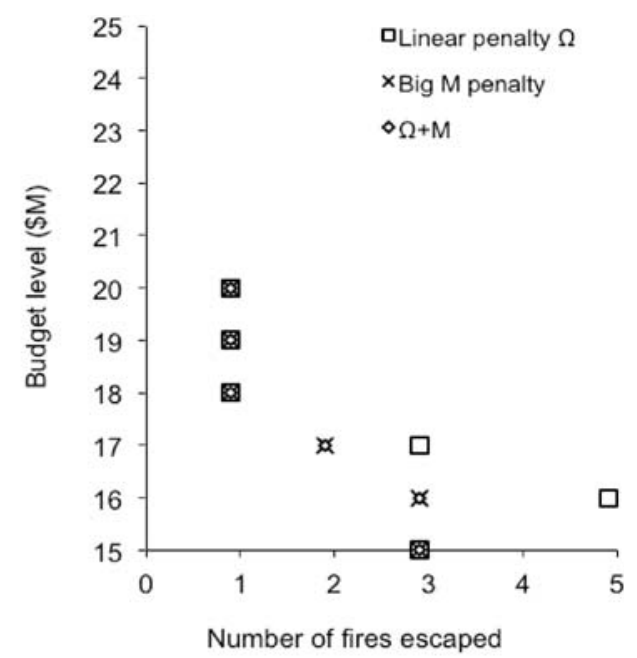

(b)

Figure 1: Fire losses within the IA duration (panel a) and number of fires escaped (panel b) across different suppression budget levels by implementing alternative penalties for escaped fire in the objective function.

By using the penalty in objective function (11), $\mathrm{F}_{9}$ would be contained because containing it will add a value of $6.7+\mathrm{M}$ (where $\mathrm{M}$ is the large constant penalty) to the objective function, which is less than adding a value of $8.4+\mathrm{M}$ to objective function from containing $\mathrm{F}_{10}$ (Table 2). This is an inferior solution because the more important fire $\left(\mathrm{F}_{10}\right)$ escaped.

Objective function (12) penalized each escape proportionate to its loss at the time of escape. Given the IA scope of the analysis, this might reflect the best, albeit imperfect, information available to the model. Weighted size reflects the last known information from IA regarding values at risk, the size of the fire, and the likely cost of managing fire in an EA setting. Here, with a budget level that is insufficient to contain all the fires, containment decisions reflect the relative importance of fires at escape. Test results, with $\Omega=1$, show that as the budget increased from $\$ 15 \mathrm{M}$ to $\$ 16 \mathrm{M}$, the number of escaped fires increased from three to five (Fig. 1b) while the loss decreased from 1,771 to 1,429 (Fig. 1a). With an addition of $\$ 1 \mathrm{M}$ to the budget, the model shifted from containing a group of five less important fires to a group of three more important fires. This local result reflects the possibility of encountering the economically inferior fire (fires that would not be contained at higher budget levels). Globally, however, as the budget increases so will the number of contained fires (Fig. 1b). In (12) the value of $\Omega$ can be increased in an attempt to reduce the number of escapes, but this is nearly always futile because increasing the value of $\Omega$ does not change the relative importance between escaped fires. Increasing $\Omega$ had no effect on containment decisions in our example.

Objective function (13) combines the costs from objective functions (11) (cost per fire) and (12) (loss at escape). By using a large constant penalty $M$ the model will contain as many fires as possible, thus maximizing initial attack success rate. If there are multiple ways of containing the same number of fires, the model will select the most important fires. For example, at budget levels from $\$ 17 \mathrm{M}$ to $\$ 20 \mathrm{M}$, it will choose $\mathrm{F}_{10}$ instead of $\mathrm{F}_{9}$ (Table 2) because $\mathrm{F}_{10}$ is 
more important (Table 2). Model results also show that by using this objective function, as the budget level increased from $\$ 15 \mathrm{M}$ to $\$ 16 \mathrm{M}$, the initial attack success rate did not decline (Fig. 1b). This suggests that objective function (12) suffers from the same problem as objective function (11), where five less important fires were contained but three more important fires are allowed to escape.

\section{CONCLUSION}

The ILP model developed in this paper includes several innovations while demonstrating key economic principles of optimal initial attack. The ILP expanded on previous work [13] to address the planning principles for a set of fires. It shows how scarce firefighting resources would be allocated to alternative fires to minimize loss at any given budget or appropriation level. By addressing the allocation of resources across a set of fires, we enabled the model to identify which fires to fight and how aggressively to fight them. In this way, the model also demonstrated how optimal dispatch locations can be scheduled and how different kinds of firefighting resources might be utilized. Altering firefighting resource scarcity through budget levels also demonstrates how optimal results and their locations are dependent upon the level of the budget. Increases in the available budget allow for greater loss reduction and usage of more effective resources, but changes in the available budget can affect optimal location decisions. The management of scarce resources is particularly important when simultaneous fire events are considered. While all fires compete for scarce resources across a planning season, simultaneous fires compete more intensively by effectively precluding the simultaneous use of individual firefighting resources. Our example showed how two simultaneous fires were managed differently by different kinds of resources to minimize overall loss.

Optimal resource use for initial attack requires that key cost elements are included in the model. These include the cost of having firefighting resources available (fixed cost), deployment costs (variable costs), and the cost of fires escaping IA. Managing the cost of escapes within the initial attack scope is inherently problematic because, by definition, they are external to the scope of analysis. Hence, they can pose the classic externality problem if not properly analyzed. Because expanding the scope of analysis to extended attack (and potentially beyond) is currently infeasible we analyzed three alternative approaches to include a proxy for this cost. The first proxy maximized initial attack success rate by including a large per fire cost where all fires escape costs were treated equally. This resulted in important fires escaping under the constrained budget while relatively unimportant fires were contained. The second proxy introduced a cost based upon the loss at the time of escape. While this approach distinguishes between important and unimportant fires, a local consequence is that fewer fires may be contained as the budget increases. The principle applied is intended to reflect the potential cost and especially cost differences of fires that would escape. The technology applied to make these cost estimates could be greatly expanded through predictive fire behavior modeling and GIS mapping to generate a reasonable estimate of escaped fire cost. However, improving the technology does not alter the principles in this demonstration. The third proxy includes both costs modeled simultaneously. Since the priority of this proxy is to maximize the IA success rate, it could also allow important fires to escape while containing fires of lesser importance.

While the ILP was intended to demonstrate managerial principles of optimal resource use in preparedness planning, especially in initial attack, it serves several other purposes. First, it is a useful demonstration of key economic elements of optimal resource allocation across a set of fires or initial attack. Such a model can also serve as a framework for thinking about how decisions can be made in ways that are consistent with principles economic 
efficiency. Secondly, an ILP model can be augmented or modified in many ways. For instance, instead of using a single fire scenario, as we did here, multiple scenarios could be used. Other enhancements could include a stochastic analysis of modeling the uncertainties in size and cost of escaped fires, and the variations existed in fire line productivities. Optimal deployment models, such as the approach illustrated here provide potentially useful insights for understanding and illustrating the efficient use of scarce resources. While optimization models have strengths and weaknesses, capitalizing on the strengths may be best realized by combining optimization with other complementary approaches such as simulation.

\section{ACKNOWLEDGEMENTS}

The authors gratefully acknowledge the support of the U.S. Department of the Intrior and of Pamela S. Zeisler, Doctoral Candidate in the Department of Forest and Rangeland Stewardship at Colorado State University.

\section{REFERENCES}

[1] United States General Accounting Office (GAO), Wildland Fire Management: Improved Planning Will Help Agencies Better Identify Fire-Fighting Preparedness Needs, Report GAO-02-158. United States General Accounting Office: Washington, D. C., 35 p., 2002.

[2] United States Government Accountability Office (GAO), Wildland Fire Management: Forest Service and Interior Need to Specify Steps and a Schedule for Identifying Long-Term Options and Their Costs, Testimony GAO-05-353T, United States Government Accountability Office: Washington, D. C., 15 p., 2005.

[3] Mills, T.J. \& Bratten, F.W., FEES: Design of a Fire Economics Evaluation System, USDA Forest Service General Technical Report No. PSW-65. 26 p., 1982.

[4] USDA Forest Service, Fire Management Analysis and Planning Handbook WO Amendment 5109.19. USDA Forest Service: Washington, D. C., 1991.

[5] Gilless, K.J. \& Fried, J.S., Stochastic representation of fire behavior in a wildland fire protection planning model for California. Forest Science., 45, pp. 492-499, 1998.

[6] McAlpine, R.S. \& Hirsch, K.G., LEOPARDS - Level of protection analysis software. Forestry Chronicle, 75, pp. 615-621, 1998.

[7] Wiitala, M.R. \& Wilson, A.E., Proceedings of the Second International Symposium on Fire Economics, Planning and Policy: A Global View. Gen. Tech. Rep. PSW-GTR-208, ed. Gonzalez-Caban, A. Pacific Southwest Research Station, Forest Service, U.S. Department of Agriculture: Albany, CA, 720 p., 2004.

[8] Parks, G.M., Development and application of a model for suppression of forest fires. Management Science, 10, pp. 760-766, 1964. doi:http://dx.doi.org/10.1287/ $\underline{\text { mnsc. } 10.4 .760}$

[9] Parlar, M. \& Vickson, R.G., Optimal forest fire control: an extension of Parks' model. Forest Science, 28, pp. 345-355, 1982.

[10] Parlar, M., Optimal forest fire control with limited reinforcements. Optimal Control Application and Methods, 4, pp. 185-191, 1983. doi:http://dx.doi.org/10.1002/ oca.4660040208

[11] Aneja, Y.P. \& Parlar, M., Optimal staffing of a forest fire fighting organization. Canadian Journal of Forest Research, 14, pp. 589-594, 1984. doi:http://dx.doi.org/10.1139/ $\underline{\mathrm{x} 84-106}$

[12] Boychuk, D. \& Martell, D.L., A Markov chain model for evaluating seasonal fores fire fighter requirements. Forest Science, 34, pp. 647-661, 1988. 
[13] Donovan, G.H. \& Rideout, D.B., An integer programming model to optimize resource allocation for wildfire containment. Forest Science, 49, pp. 331-335, 2003.

[14] Haight, R.G. \& Fried, J.S., Deploying wildland fire suppression resources with a scenario-based standard response model. Information Systems and Operational Research, 45(1), pp. 31-39, 2007. doi:http://dx.doi.org/10.3138/infor.45.1.31

[15] Bevers, M., A chance contraint estimation approach to optimizing resource management under uncertainty. Canadian Journal of Forest Research, 37(11), pp. 2270-2280, 2007. doi:http://dx.doi.org/10.1139/X07-076

[16] Rideout, D.B. \& Omi, P.N., Alternate expressions for the economic theory of forest fire management. Forest Science, 36(3), pp. 614-624, 1990.

[17] Rideout, D.B. et al., Estimating rates of substitution for protecting values at risk for initial attack planning and budgeting. Forest Policy and Economics, 10(2), pp. 205-219, 2008. doi:http://dx.doi.org/10.1016/j.forpol.2007.10.003

[18] Bradley, M. et al., The national wildfire prediction program: a key piece of the wildfire solution. The Joint Fire Science Conference and Workshop: Boise, ID, 2000.

[19] Miller, C., Davis, B., \& Black, A., Using the probability of burning to plan for wildland fire use. 2nd International Wildland Fire Ecology and Fire Management Congress, Orlando, FL, 2003.

[20] Prestemon, J.P. et al., Understanding broad-scale wildfire risks in a human-dominated landscape. Forest Science, 48, pp. 685-693, 2002.

[21] Westerling, A.L. et al., Long lead statistical forecasts of western U.S. wildfire area burned. International Journal of Wildland Fire, 11(3,4), pp. 257-266, 2002.

[22] Bureau of Land Management (BLM), Interagency standards for fire and aviation operations, BLM Handbook 9213-1, 2003.

[23] Coase, R.H., The problem of social cost. Journal of Law and Economics, 34, pp. 1-44, 1960. doi:http://dx.doi.org/10.1086/466560

[24] Mees, R.M., Simulating initial attack with two fire containment models. USDA Forest Service Research Note PSW-378. 7 p., 1985.

[25] National Wildfire Coordinating Group (NWCG), Fireline Handbook, NWCG Handbook 3, PMS410-1, NFES 0065, Boise, ID, 1998. 